\title{
Influencia del Diámetro y Longitud de Implantes en la Pérdida Tardía de Implantes Dentales
}

\author{
Influence of Diameter and Length of Implant on Late Dental Implant Failure \\ Rafael Ortega Lopes*; Sergio Olate**; Claudio Ferreira Noia*; \\ Henrique Duque de Miranda Chaves Netto ${ }^{* * *}$; Marcio de Moraes* \& Renato Mazzontetto*
}

ORTEGA-LOPES, R.; OLATE, S.; NOIA, C. F.; CHAVES NETTO, H. D. M.; MORAES, M. \& MAZZONETO, R. Influencia del diámetro y longitud de implantes en la pérdida tardía de implantes dentales. Int. J. Odontostomat., 6(1):77-80, 2012.

RESUMEN: El objetivo de esta investigación fue describir la influencia del diámetro y largo de los implantes en la pérdida tardía del implante. Se diseñó un estudio retrospectivo para estudiar 375 pacientes que habían sido sometidos a rehabilitación oral sobre implantes durante un periodo de 11 años; se incluyeron en el estudio todos aquellos documentos debidamente completados y con estudios radiográficos preoperatorios, posterior a la instalación del implante y posterior a la instalación de la prótesis. Los implantes fueron clasificados de acuerdo al diámetro como estrecho, regular y ancho y de acuerdo al largo como corto, medio y largo; el análisis de datos fue realizado con la prueba Chi Cuadrado con valor de $p<0,05$. Del total de 939 implantes y se identificó la pérdida de 55 implantes posterior a la etapa protésica (5,8\%). En base a la pérdida, no se logró establecer ninguna relación estadísticamente significativa con el diámetro del dispositivo $(p=0,475)$ y tampoco con el largo del implante $(p=0,064)$. Podemos concluir que el largo y el diámetro de implantes dentales no influyen en la pérdida tardía del mismo.

PALABRAS CLAVE: implante dental, pérdida tardía, sobrevida de implantes.

\section{INTRODUCCIÓN}

La diversidad de rehabilitaciones protésicas implanto asistidas se ha logrado, entre otras cosas, por la variabilidad de diseños de implantes y sus diferentes largos y anchos (Misch, 1990; Starr, 2001). Las limitaciones anatómicas del reborde alveolar y la presencia de estructuras nobles como el seno maxilar y el nervio alveolar inferior también han influido en el desarrollo de modificaciones de largo y ancho de implantes (Langer et al., 1993; Spierkemann et al., 1995).

En términos de éxito del tratamiento, algunos autores sostienen que el uso de implantes de entre 10 $\mathrm{mm}$ y $13 \mathrm{~mm}$ de largo presenta los mejores indicadores de sobrevida (Goodacre et al., 2003; Eckert et al., 2001) y cuando estos son asociados a diámetros regulares (4,0 $\mathrm{mm}$ aproximadamente) y con tratamiento de superficie esta sobrevida es aún mayor (Goodacre et al., 1999; Teixeira et al., 1997).
De esta forma, el fracaso del tratamiento con implantes dentales puede ser asociado a la curva de aprendizaje del cirujano y rehabilitador, la estabilidad primaria deficiente, el tipo de superficie del implante, la cantidad y calidad ósea, entre otros (Tawill \& Younan, 2003).

En cuanto al diámetro de implantes, los resultados expuestos han sido variados e inicialmente controversiales; algunos autores han señalado el éxito de los implantes anchos y otros han observaron resultados contrarios (Tada et al., 2003; Olate et al., 2010). A pesar que el manejo bioquímico (tratamiento y manejo de superficie) de los implantes y su relación con el tejido duro y blando han mejorado con el desarrollo de la tecnología (Chun et al., 2002; Tada et al.) y la experiencia quirúrgica y protésica han mejorado, aun es posible observar dudas respecto del éxito de im-

\footnotetext{
- División de Cirugía Oral y Maxilofacial, Universidad Estadual de Campinas, Brasil

" Unidad de Cirugía Oral y Maxilofacial, Universidad de La Frontera, Chile.

${ }^{* * *}$ División de Cirugía Oral y Maxilofacial, Universidad Federal de Juiz de Fora, Brasil.
} 
plantes de diámetros y el largos extremos después de que la rehabilitación protésica ha sido realizada. De esta forma, el objetivo de esta investigación es reconocer la pérdida de implantes posterior a la instalación de la rehabilitación protésica y relacionarlos con el ancho y largo de los dispositivos perdidos.

\section{MATERIAL Y MÉTODO}

Se diseñó un estudio retrospectivo para el análisis de 375 pacientes operados en el Servicio de Cirugía Oral y Maxilofacial de la Facultad de Odontología de Piracicaba de la Universidad Estadual de Campinas (Brasil) durante un period de 11 años. La investigación fue aprobada por el Comité de Ética en investigación CEP-FOP/UNICAMP 002/2007. Criterios de inclusión fueron que fichas clínicas presentaran todos los datos necesarios para la evaluación junto a los estudios de imagen realizados en la etapa prequirúrgica, postquirúrgica y posterior a la instalación de la prótesis. Todos los archivos incompletes o con instalación del sistema protésico en otros servicios, tanto públicos como privados, fueron excluidos del estudio. Todas las cirugías fueron realizadas por residentes del cirugía oral y maxilofacial y por residentes de implantología.

La pérdida tardía de implantes fue estudiada a partir del primer mes posterior a la instalación de la prótesis; las variables asociadas al implante fueron establecidas en base al diámetro, siendo de tipo estrecho (3,3 a 3,5 mm de diámetro), regular (3,75 a 4,3 $\mathrm{mm}$ ) y anchos (mayors que $5 \mathrm{~mm}$ ). En relación al largo, los implantes fueron clasificados como cortos (menores que $8,5 \mathrm{~mm})$, medianos $(8,5 \mathrm{a} 11,5 \mathrm{~mm}$ ) y largos (mayores que 11,5 mm). La pérdida de implantes fue establecida mediante el estudio clínico del paciente y el seguimiento a través de la ficha clínica.

Las variables estipuladas fueron analizadas con la prueba estadística Chi Cuadrado considerando un valor de $p<0,05$, para lo cual fue utilizado el software estadístico S.A.S. 8.2.

\section{RESULTADOS}

Fueron estudiados un total de 375 pacientes, siendo 234 pertenecientes al sexo femenino $(62,4 \%)$ y 141 al sexo masculino $(37,6 \%)$. La edad de los pa- cientes fluctuaba entre 17 y 86 años con una edad promedio de 48,9 años. De los 939 implantes, en maxila fueron instalados 412 implantes (43,8\%), mientras que en mandíbula fueron 527 implantes $(56,1 \%)$.

Las rehabilitaciones instaladas en los implantes fueron de tipo fija, unitaria o múltiple, en 749 implantes $(79,7 \%)$, mientras que en 190 implantes se rehabilitó con prótesis removible $(20,3 \%)$. Los tipos de conexión y retención protésica fueron cementadas en 662 implantes $(70,5 \%)$, atornilladas para rehabilitación unitaria o doble en 72 implantes $(7,7 \%)$, atornilladas con sistema o'ring en 141 implantes (15\%) y atornilladas con barra de retención en 64 implantes $(6,8 \%)$.

La evaluación de la pérdida de los implantes fue realizada a partir del primer mes posterior a la instalación de la prótesis, observándose las pérdida de implantes entre el primer mes y el quinceavo mes posterior a la instalación de la prótesis sobre el implante.

De los 939 implantes fueron instalados $11 \mathrm{im}$ plantes de diámetro estrecho (1,2\%), 752 implantes de diámetro regular $(80 \%)$ y 176 implantes anchos $(18,8 \%)$. En base al largo fueron instalados 8 implantes cortos $(0,9 \%), 457$ implantes medianos $(48,6 \%)$ y 474 implantes largos (50,5\%).

La pérdida tardía se observó en 55 implantes (5,8\%), siendo ellos 42 implantes de diámetro regular $(76,4 \%)$ y 13 de diámetro mayor a $4,3 \mathrm{~mm}(23,6 \%)$; no se observó relación estadísticamente significativa para esta variable $(p=0,475)$. En relación al largo del implante, 35 fueron de tipo regular (64\%) y 20 fueron implantes largos (36\%) y no se observó relación estadísticamente significativa entre la pérdida tardía del implante y el largo del mismo $(p=0,064)$

\section{DISCUSIÓN}

Los datos aportados por el presente estudio son clarificadores, presentando un éxito cercano al 94,5\% después de la carga protésica del implante.

Largo del implante. En algunos estudios se ha observado la reducción de la sobrevida de los implantes cortos, determinando además que el diámetro aumentado es más complejo para la sobrevida de los implantes que el largo del mismo (Block et al., 1990; Goodacre et al., 1999; Teixeira et al.; Bahat, 1993). 
Implantes más largos que $13 \mathrm{~mm}$ no presentan mayor sobrevida o mejores indicadores de éxito al ser comparado con implantes de largo regular (Eckert et al.; Goodacre et al., 2003). La incorrecta indicación, el trabajo en hueso deficiente y la curva de aprendizaje fueron causas importantes para la pérdida de implantes cortos (Renouard \& Rangert, 2008).

Del punto de vista biomecánico existe consenso en que el mayor largo no es sinónimo de mayor rendimiento clínico; de hecho, en análisis por elementos finitos se observó que las presiones por cizallamiento con fuerzas oblicuas se concentran en los primeros $7 \mathrm{~mm}$ del implante sin diferencias asociadas al largo del implante (Pierrisnard et al., 2000). Por otra parte, si consideramos que la pérdida ósea normal a nivel cervical es cercana a los $0,2 \mathrm{~mm}$ al año (Misch; Langer et al.; Spiekermann et al.), es posible esperar que implantes cortos presenten menos contacto óseo al pasar de los años.

Diámetro de implantes. Al analizar el diámetro de implantes, la literatura científica es controversial. En algunas investigaciones, el implante de diámetro regular presenta $5 \%$ más de sobrevida al compararse con implantes de diámetro reducido (Spikermann et al.; Starr). Uno de los mayores indicadores de fracaso para implantes anchos (cercano al 18\%) fue presentado por Ivanoff et al. (1999), probablemente debido a que siendo un estudio retrospectivo, aún no presentaba la evolución de la técnica y los materiales, la indicación de instalación era principalmente como implante de rescate y la ascendente curva de aprendizaje se encontraba en sus etapas iniciales. A partir del año 2000 , Polizi et al. sugerían que mientras mayor fuese el diámetro del implante, mayor sería la superficie de contacto y por lo tanto mayores posibilidades de éxito del tratamiento (Polizi et al., 2000); si bien es real el aumento de la superficie de contacto, no debemos olvidar que en estos dispositivos existe menos contacto con tejido óseo esponjoso y mayor contacto con tejido oseo cortical, por definición menos vascularizado, lo cual podría alterar la oseintegración del dispositivo (Tada et al.; Olate et al.).

Del punto de vista biomecánico, Vigolo \& Givani (2000) determinaron que implantes estrechos presentan una resistencia a la fractura $25 \%$ menor que los implantes de diametro regular. Los estudios retrospectivos muestran índices de fracaso del 5\% a $7 \%$ mayor a los implantes regulares (Spikermann et al.; Starr).

Se ha señalado que algunas causas para la falla de los implantes anchos ha sido la pobre calidad ósea (mayor proporción de hueso cortical en contacto con el implante), la instalación en lugares donde previamente hubo otro implante, calentamiento inadecuado del hueso en la etapa de preparación del lecho, compresión excesiva del hueso en la instalación del implante (Shin et al., 2004; Davarpanah et al., 2001; Lee et al., 2005), entre otros. Modificaciones en la secuencia de fresado y el uso de técnicas como las de osteotomos permiten mayor conservación ósea y el aumento del éxito del tratamiento

El bajo numero de implantes estrechos presentes en este estudio puede justificar la ausencia de relaciones estadísticas entre las variables estudiadas. Esto principalmente porque en nuestra vision de tratamiento, cuando existen deficiencias del ancho óseo se indica la reconstrucción ósea previa con injerto óseo autógeno (Mazzonetto, 2008; Nóia, 2011) y no realizamos esfuerzos heroicos por intentar instalar implantes estrechos en lugares con deficiencia ósea.

En base a nuestra muestra de estudio, podemos concluir que los factores relacionados con el largo y ancho del implante no influyen en la pérdida tardía del mismo.

ORTEGA-LOPES, R.; OLATE, S.; NOIA, C. F.; CHAVES NETTO, H. D. M.; MORAES, M. \& MAZZONETO, R. Influence of diameter and length of implant on late dental implant failure. Int. J. Odontostomat., 6(1):77-80, 2012.

ABSTRACT: The aim of this research was to describe the influence of diameter and length of dental implant related to late implant faliure. In a retrospective study was evaluated 375 patients in a 11 year period; were inclued the complet medical record with image study in the preoperative period, posteriorly to implant installation and to prosthesis installation. The implant was classified according to diameter in narrow, regular and wide, and according to length as short, regular and long. The statistical analysis was realized with Chi-Squaare test with $p$ value $<0.05$. We studied 939 implants and observed 55 implant failure posteriorly to prosthesis phases $(5.8 \%)$. No statistical relation with diameter $(p=0.475)$ or lenght $(p=0.064)$ was observed. We conclude that the length and diameter of dental implant is not an influence for late failure of the implant. 


\section{REFERENCIAS BIBLIOGRÁFICAS}

Bahat, O. Treatment planning and placement of implants in the posterior maxillae: report of 732 consecutive Nobelpharma implants. Int. J. Oral Maxillofac. Implants, 8(2):151-61, 1993.

Block, M. S.; Delgado, A. \& Fontenot, M. The effect of diameter and length of hydroxylapatite-coated dental implants on ultimate pullout force in dog alveolar bone. J. Oral. Maxillofac. Surg., 48(2):174-8, 1990

Chun, H. J.; Cheong, S. Y.; Han, J. H.; Heo, S. J.; Chung, J. P.; Rhyu, I. C.; Choi, Y. C.; Baik, H. K.; Ku, Y. \& Kim, M. H. Evaluation of design parameters of osseointegrated dental implants using finite element analysis. J. Oral Rehabil., 29(6):565-74, 2002.

Davarpanah, M.; Martinez, H.; Tecucianu, J. F.; Alcoforado, G.; Etienne, D. \& Celletti, R. The self-tapping and ICE 3i implants: a prospective 3-year multicenter evaluation. Int. J. Oral Maxillofac. Implants, 16(1):52-60, 2001.

Eckert, S. E.; Meraw, S. J.; Weaver, A. L. \& Lohse, C. M. Early experience with Wide-Platform Mk II implants. Part I: Implant survival. Part II: Evaluation of risk factors involving implant survival. Int. J. Oral Maxillofac. Implants, 16(2):208-16, 2001.

Goodacre, C. J.; Bernal, G.; Rungcharassaeng, K. \& Kan, J. Y. Clinical complications with implants and implant prostheses. J. Prosthet. Dent., 90(2):121-32, 2003.

Goodacre, C. J.; Kan, J. Y. \& Rungcharassaeng, K. Clinical complications of osseointegrated implants. J. Prosthet. Dent., 81(5):537-52, 1999

Ivanoff, C. J.; Gröndahl, K.; Sennerby, L.; Bergström, C. \& Lekholm, U. Influence of variations in implant diameters: a 3- to 5-year retrospective clinical report. Int. J. Oral Maxillofac. Implants, 14(2):173-80, 1999.

Langer, B.; Langer, L.; Herrmann, I. \& Jorneus, L. The wide fixture: a solution for special bone situations and a rescue for the compromised implant. Part1. Int. J. Oral Maxillofac. Implants, 8(4):400-8, 1993.

Lee, J. H.; Frias, V.; Lee, K. W. \& Wright, R. F. Effect of implant size and shape on implant success rates: a literature review. J. Prosthet. Dent., 94(4):377-81, 2005.

Mazzonetto, R. Reconstruções em implantodontia. Protocolos clínicos para o sucesso e previsibilidade. Nova Odessa, Napoleão, 2008.

Misch, C. E. Divisions of available bone in implant dentistry. Int. J. Oral Implantol., 7(1):9-17, 1990.

Nóia, C. F. Avaliação radiográfica prospectiva de alterações estéticas em pacientes submetidos à remoção de enxertos de mento. Tese mestrado. Piracicaba, Programa de PósGraduação em Clínica Odontológica, Universidade Estadual de Campinas, 2011.
Olate, S.; Lyrio, M. C.; de Moraes, M.; Mazzonetto, R. \& Moreira, R. W. Influence of diameter and length of implant on early dental implant failure. J. Oral Maxillofac. Surg., 68(2):414-9, 2010.

Pierrisnard, L.; Augereau, D. \& Barquins, M. Analyse comparative par la méthode des elements finis des contraintes osseuses induites par des implants de géométrie variée. 2. Influence relative de la longueur et de lórientation des implants. Implants, 6:93-105, 2000.

Polizzi, G.; Rangert, B.; Lekholm, U.; Gualini, F. \& Lindstrom, H. Brånemark System Wide Platform implants for single molar replacement: clinical evaluation of prospective and retrospective materials. Clin. Implant Dent. Relat. Res., 2(2):61-9, 2000.

Renouard, F. \& Rangert, B. Fatores de risco em implantodontia: Análise clínica simplificada para um tratamento previsível. São Paulo, Quintessence Editora Ltda., 2008.

Shin, S. W.; Bryant, S. R. \& Zarb, G. A. A retrospective study on the treatment outcome of wide-bodied implants. Int. J. Prosthodont., 17(1):52-8, 2004.

Spiekermann, H.; Jansen, V. K. \& Richter, E. J. A 10-year followup study of IMZ and TPS implants in the edentulous mandible using bar-retained overdentures. Int. J. Oral Maxillofac. Implants, 10(2):231-43, 1995.

Starr, N. L. The distal extension case: An alternative restorative design for implant prosthetics. Int. J. Periodontics Restorative Dent., 21(1):61-7, 2001.

Tada, S.; Stegaroiu, R.; Kitamura, E.; Miyakawa, O. \& Kusakari, $\mathrm{H}$. Influence of implant design and bone quality on stress/ strain distribution in bone around implants: a 3-dimensional finite element analysis. Int. J. Oral Maxillofac. Implants, 18(3):357-68, 2003.

Tawil, G. \& Younan, R. Clinical evaluation of short, machinedsurface implants followed for 12 to 92 months. Int. J. Oral Maxillofac. Implants, 18(6):894-901, 2003.

Teixeira, E. R.; Wadamoto, M.; Akagawa, Y. \& Kimoto, T. Clinical application of short hydroxylapatite-coated dental implants to the posterior mandible: a five-year survival study. J. Prosthet. Dent., 78(2):166-71, 1997.

Vigolo, P. \& Givani, A. Clinical evaluation of single-tooth miniimplant restorations: a five-year retrospective study. J. Prosthet. Dent., 84(1):50-4, 2000.

Dirección para correspondencia:

Rafael Ortega Lopes

Faculdade de Odontologia de Piracicaba - FOP/Unicamp

Avenida Limeira, 901 Bairro: Areião

CEP: $13.414-903$

Piracicaba - São Paulo

BRASIL

Recibido : 02-11-2011

Aceptado: 03-12-2011

E-mail: rafaellopes@fop.unicamp.br 